\title{
DESENVOLVIMENTO DE UM MODELO MATEMÁTICO DO PROBLEMA DE ROTEAMENTO DE VEÍCULOS COM FROTA HETEROGÊNEA
}

\author{
Mateus de Assis Chacon Lima \\ Universidade Federal de Uberlândia - UFU \\ mateusdeassislima@gmail.com \\ Rafael Hiroshi Harada Soares \\ Universidade Federal de Uberlândia - UFU \\ rhirosh@gmail.com \\ Jorge von Atzigen dos Reis \\ Universidade Federal de Uberlândia - UFU \\ jorgereis@ufu.br \\ Marcos Caixeta Valim \\ Latu \\ caixeta@latu.com.br
}

\begin{abstract}
RESUMO
Para manter o nível de competitividade as organizações buscam meios de otimização para as operações, possibilitando o estudo e desenvolvimento de modelos de otimização, onde o roteamento de veículos se enquadra nos problemas clássicos dentro do gerenciamento logístico. O presente trabalho aborda um estudo onde é necessário desenvolver e implementar um modelo matemático capaz de determinar rotas para diferentes veículos com capacidades distintas, o mesmo foi proposto por uma empresa de software logístico. O estudo é baseado na formulação do modelo matemático, a utilização do software de otimização para resolução do modelo e análise dos resultados obtidos, com intuito de buscar a minimização de custos da operação logística, ou seja, custos referentes ao frete e a geração da ordem de rotas, onde foi obtido bons resultados para uma quantidade limitada da frota de veículos e pontos de coletas.
\end{abstract}

Palavra-chave: Otimização; Problema do Roteamento de Veículo; Modelagem Matemática.

\begin{abstract}
$\mathrm{n}$ order to maintain the level of competitiveness, organizations are looking for ways to optimize operations, enabling the study and development of optimization models, where vehicle routing fits into the classic problems within logistics management. The present work addresses a study where it is necessary to develop and implement a mathematical model capable of determining routes for different vehicles with different capacities, the same was proposed by a logistics software company. The study is based on the formulation of the mathematical model, the use of optimization software for model resolution and analysis of the results obtained, aiming to minimize the costs of logistics operation, ie costs related to freight and order generation. routes, where good results were obtained for a limited amount of vehicle fleet and collection points.
\end{abstract}

Keywords: Otimization; Vehicle Routing Problem; Mathematical Modeling. 


\section{Como Citar:}

LIMA, Mateus de Assis Chaconet et al. Desenvolvimento de um modelo matemático do problema de roteamento de veículos com frota heterogênea. In: SIMPÓSIO DE PESQUISA OPERACIONAL E LOGÍSTICA DA MARINHA, 19., 2019, Rio de Janeiro, RJ. Anais [...]. Rio de Janeiro: Centro de Análises de Sistemas Navais, 2019.

\section{INTRODUÇÃO}

Atualmente as operações logísticas possuem uma grande importância no planejamento corporativo das empresas, isso se justifica em algumas operações, em que o custo e o planejamento envolvido são complexos e significantes para a corporação, pois o nível de serviço prestado deve ser compatível com a necessidade do mercado atual, que por sua vez está dinâmico e exigente (ARAÚJO, 2017). Desta forma é necessário mensurar o equilíbrio entre o nível de serviço e os custos envolvidos nas operações logísticas, sejam elas no transporte ou no armazenamento de bens.

Dentro deste cenário a Latu - empresa especializada em soluções de tecnologia e otimização no setor de transportes - propôs um desafio para estudo e criação de um caso para gerenciamento de e geração de rotas para frota heteroĝenea de veículos, tendo capacidade individual de carga e custo de frete, para captação de produtos agrícolas em zonas rurais com pontos fixos de coleta. A Latu Negócios é uma empresa de tecnologia fundada em 2009 com foco no setor de transportes desde 2015, com experiência no desenvolvimento de sistema de otimização de fiscalização em documentos de transportadoras , realiza consultorias para habilitação de empresas de meios de pagamentos para operar atividades de vale pedágio obrigatório e pagamento eletrônico de frete e está, através dos conceitos levantados neste trabalho, implementando um novo produto para otimização de coleta e entrega de produtos no setor agro.

Definindo o problema e sendo perceptível a complexidade de geração de rotas, a Pesquisa Operacional possibilita a aplicação de ferramentas para o estudo e desenvolvimento de modelos matemáticos para resolução, que é caracterizado como um problema clássico de Vehicle Routing Problem - VRP, segundo Arenales (2007) esse modelo apresenta a existência de uma frota de veículos que devem atender um conjunto de clientes de forma a captar seus recursos e entregar em um ponto de destino que geralmente é nas indústrias, levando em consideração que a geração da ordem de rotas tenha a menor distância possível.

A linha de raciocínio para desenvolvimento deste presente trabalho é formular um modelo matemático para o problema proposto, a implementação do algoritmo em um software de otimização e a realização de teste para analisar o comportamento do tempo de resolução e capacidade de memória RAM da máquina de teste. Utilizando como base de testes hipóteses com diferentes quantidades de pontos a serem visitados assim como o tamanho da frota de veículos.

\section{REFERENCIAL TEÓRICO}

Nesta seção foi realizado um levantamento bibliográfico sobre logística, especificamente sobre modelos de roteirização com frota homogênea, pois a proposta do presente trabalho é criar uma formulação matemática para frota heterogênea se baseando na formulação clássica.

\subsection{LOGÍSTICA}

De acordo com Bowersox (2007), a logística é estudo científico através do planejamento, onde as operações logísticas devem ser feitas de acordo com tempo, 
quantidades e destinos planejados, sempre buscando o menor custo e o máximo nível de serviço. Em algumas operações o fluxo de informação do que está acontecendo ou que já aconteceu é importante para manutenção e controle do planejamento logístico. As maiores dificuldades no planejamento envolvem questões de quantidade de cliente, armazenamento de estoque, custos referentes as distâncias, decisões de rotas, quantidades de ativos de transporte e capacidade dos ativos.

Dessa forma Mentzer (2008), aborda a dificuldade em definir a cadeia de suprimentos que complementa a logística, com o planejamento e gerenciamento de todas as atividades envolvidos em abastecimentos e aquisições. Incluindo coordenação e colaboração com parceiros como fornecedores, provedores de serviços terceirizados e clientes.

Dentro do planejamento logístico a roteirização é uma ferramenta que busca direcionar as operações logísticas, ou seja, ativos de transportes são designados para melhor atender os clientes buscando sempre o nível de serviço que os atenda melhor, esse método pode ser realizado através de técnicas específicas que são processos clássicos desenvolvido por algum grupo de pessoas ou por softwares que conseguem fazer automaticamente. Segundo Taha (2008), essa atividade tem como princípio determinar roteiros, paradas e sequências, buscando a minimização de tempos, rotas e consequentemente custos, sempre atendendo a todos os locais pré-determinados.

\subsection{PESQUISA OPERACIONAL - VRP}

Um dos problemas clássicos dentro da pesquisa operacional e no gerenciamento logístico é o roteamento de veículos, conhecido também como Vehicle Routing Problem ou simplesmente VRP, onde o mesmo baseia-se na construção de rotas para cada caminhão, atendendo todos os pontos solicitados no problema. Esse tipo de modelo geralmente é utilizado nos projetos de rotas para entrega ou coleta. Na formulação clássica proposta pelo Arenales (2007), o VRP é representado por um conjunto de grafos orientados e completos G $=(\mathrm{N}, \mathrm{E})$ em que $\mathrm{N}=\mathrm{C} \cup[0, \mathrm{n}+1]$, sendo que $\mathrm{C}=1, \ldots, \mathrm{n}$ é o conjunto de nós que representam os pontos a serem atendidos, 0 a origem e $n+1$ são os nós que representam $o$ depósito. $O$ conjunto $E=[(i, j) \vee i, j \in N, i \neq j, i \neq n+1, j \neq 0]$, sendo $K$ o conjunto de veículos disponíveis de uma frota homogênea. O objetivo proposto é minimizar os custos totais percorridos, atentando que cada rota deve ser iniciada no ponto 0 e terminada no ponto $\mathrm{n}+1$, os pontos serão atendidos somente uma única vez e a demanda total presente nos mesmos não pode ultrapassar a capacidade do caminhão, assim como o tempo de viagem total da rota, considerando:

- $C_{i j}$ sendo custo do veículo ao percorrer o $\operatorname{arco}(i, j)$;

- $D_{i}$ é a demanda do cliente $i$;

- $Q$ é a capacidade de cada veículo k, com frota Homogênea;

- $S$ é um conjunto de subconjunto contido dentro $\mathrm{G}=(\mathrm{N}, \mathrm{E})$;

- Variáveis de decisão $x_{i j k}$ :

- 1 se o veículo $K$ percorre o arco $(i, j), \forall k \in K, \forall(i, j) \in \mathrm{E}$;

- 0 caso contrário;

$$
\begin{gathered}
\min \sum_{k \in K} \sum_{(i, j) \in C} c_{i j} x_{i j k} \\
\sum_{k \in K} \sum_{j \in C} x_{i j k}=1 \quad \forall i \in C \\
\sum_{i \in C} d_{i} \sum_{j \in C} x_{i j k} \leq Q \quad \forall k \in K \\
\sum_{j \in C} x_{0 j k}=1 \quad \forall k \in K
\end{gathered}
$$




\section{SPOLM2019}

XIX SIMPÓSIO DE PESQUISA OPERACIONAL ELOGÍSTICA DA MARINHA

$$
\begin{gathered}
\sum_{i \in C} x_{i h k}-\sum_{j \in C} x_{h j k}=0 \quad \forall h \in C, \forall k \in K \\
\sum_{i \in C} x_{i, n+1, k}=1 \forall k \in K \\
\sum_{i \in S} \sum_{j \in S} x_{i j k} \leq|S|-1 \subset C, 2 \leq|S| \leq\left|\frac{n}{2}\right|, \forall k \in K \\
x_{i j k} \in[0,1] \forall i, j, k
\end{gathered}
$$

As equações 2.1 a 2.8 apresentam o modelo matemático para o VRP proposto em Arenales (2007).

- A equação 2.1 remete aos custos envolvidos para realização da rota percorrida de todos veículos utilizados;

- A equação 2.2 garante que todos os pontos sejam visitados

- A equação 2.3 garante que a capacidade de nenhum veículo seja excedida; $\mathrm{i}=0$;

- A equação 2.4 garante todos os veículos partam do ponto de origem considerando

- A equação 2.5 garante que os veículos k que chegam no ponto de origem, são os mesmos que partem para o ponto de destino;

- A quinta equação 2.6 garante que todos os veículos escolhidos cheguem no ponto destino, $\mathrm{i}=\mathrm{N}$;

- A equação 2.7 elimina a possibilidade da geração de sub-rota;

\section{APLICAÇÃO EMPÍRICA}

O problema foi proposto pela empresa de software logístico, onde o caso prático foi realizar o roteamento de veículos na região com frota heterogênea, sabendo que, existe uma certa quantidade de lugares a ser visitado para a coleta do produto a ser entregue em um único ponto específico, no caso a indústria.

O principal objetivo é a escolha dos caminhões e gerar rotas otimizadas considerando o menor custo por quilômetro rodado que atende toda oferta disponível na região, a frota existente para o estudo tem capacidades diferentes assim como seus custos.

\subsection{IMPLEMENTAÇÃO COMPUTACIONAL}

Considerando o problema descrito anteriormente, utilizou-se um modelo clássico de Vehicle Routing Problem - VRP, contudo não descreve em sua totalidade às necessidades reais exigidas, porém serve como referência para o desenvolvimento do estudo abordado adaptando o mesmo para se encaixar às necessidades do problema. A sequência das atividades foram do desenvolvimento do novo modelo matemático, implementação do algoritmo para o modelo e análise da fase de testes.

O software escolhido para implementação do modelo foi o GUROBI@ versão 8.1.0, por apresentar uma licença acadêmica que suporta a resolução do mesmo, a máquina de teste apresenta as seguintes especificações: processador Intel Xeon E5-1620 v2 10 M, 3,70 GHz, e possuindo $40 \mathrm{~GB}$ de memória RAM. A base de teste utilizado para o modelo foi de três instâncias de testes de possíveis hipóteses fornecidos pela empresa:

- Dispondo de 10 pontos incluindo a saída de um depósito e chegada ao destino com uma frota heterogênea de 5 caminhões;

- Dispondo de 13 pontos incluindo a saída de um depósito e chegada ao destino com uma frota heterogênea de 8 caminhões;

- Dispondo de 18 pontos incluindo a saída de um depósito e chegada ao destino com uma frota heterogênea de 8 caminhões.

Dentro de cada instância de teste existe a necessidade de gerar rotas atendendo todos pontos de coleta, a escolha das mesmas é basicamente um acréscimo de pontos e 
caminhões de forma crescente para analisar de forma sistemática o comportamento do modelo, ou seja, análise de tempo de resolução e capacidade de memória RAM. Para cada instância será criado um arquivo de entrada para alimentar o algoritmo de otimização, onde o mesmo apresenta informações de: quantidade de clientes a serem visitados, quantidade de caminhões disponíveis, capacidade de cada caminhão, custo por quilômetro para cada caminhão e a matriz distância de todos os pontos em quilômetros.

\subsection{Modelagem Matemática}

A disposição do problema de roteamento do veículo para captação e circulação é um elemento-chave para determinar o padrão da coleta e do ciclo de fornecimento de veículos. A organização do caminho da captação é benéfica para economizar tempo, redução de custos de transportes, melhoria da eficiência e a qualidade do nível de serviço. Considerando as informações necessárias para as empresas de logística que usam múltiplos veículos para a coleta de produtos, a localização de cada fornecedor, o volume da carga proposta e o volume total que cada veículo suporta, são informações constantes, assegurando que o custo total seja mínimo. Considerando o modelo clássico proposto em Arenales (2007), e modificando as restrições para adequar a frota heterogênea e as escolhas dos caminhões, segue os parâmetros para configuração do VRP proposto no presente artigo:

- $C_{k}$ sendo custo do veículo $k$;

- $D_{i j}$ é a distância do fornecedor $i$ para o fornecedor $j$;

- Capacidade $_{k}$ é a capacidade do veículo $k$;

- $\quad$ Demanda $_{i}$ a demanda do fornecedor $i$;

- $S$ é um conjunto de subconjunto contido dentro de N.

- Variáveis de decisão $x_{k i j}$ :

- 1 , caso veículo k conduz do fornecedor $i$ para o fornecedor $j$;

- 0 , caso contrário.

- Variáveis de decisão $y_{k}$ :

- 1 , caso veículo $\mathrm{k}$ for utilizado;

- 0, caso contrário.

$$
\begin{gathered}
\min Z=\sum_{k \in K} \sum_{i \in C} \sum_{j \in C} x_{k i j} D_{i j} C_{k} \\
\sum_{k \in K} \sum_{j \in C} x_{i j k}=1 \quad \forall i \in C \neq 1 \\
\sum_{i \in C} x_{k i h}-\sum_{j \in C}^{j} x_{k h j}=0 \quad \forall h \in C, \forall k \in K \\
\sum_{j \in C} x_{k 0 j}=y_{k} \forall k \in K \\
\sum_{i \in C}^{n} x_{k i N}=y_{k} \forall k \in K \\
\sum_{i \in C} \sum_{j \in C} \operatorname{Demanda}_{i} x_{i j k} \leq \text { Capacidade } y_{k} \forall k \in K \\
\sum_{i \in S} \sum_{j \in S} x_{k i j} \leq|S|-1 \subset C, 2 \leq|S| \leq\left|\frac{n}{2}\right|, \forall k \in K \\
x_{k i j} \in[0,1] \forall i, j, k \\
y_{k} \in[0,1] \forall k
\end{gathered}
$$

- A equação 3.1 Função objetivo remete ao custo total envolvido para realização da rota percorrida de todos veículos utilizados;

- A segunda equação 3.2 considera que cada veículo é obrigado a passar em todos 
os pontos exceto nos pontos de origem e destino;

- A terceira equação 3.3 garante que os veículos $k$ que chegam no ponto de origem são os mesmos que partem para o ponto de destino;

- A quarta equação 3.4 garante que todos os veículos escolhidos saiam do depósito, ponto de origem, $\mathrm{i}=0$;

- A quinta equação 3.5 garante que todos os veículos escolhidos cheguem na indústria, ponto destino, $\mathrm{i}=\mathrm{N}$;

- A sexta equação 3.6 garante que todos os veículos escolhidos, não seja esgotada sua capacidade recolhendo a demanda nos arcos (i, j);

- A sétima equação 3.7 elimina a possibilidade da realização de sub-rotas dos veículos k, essa estrição é conjunto de subconjuntos presente dentro do conjunto N_cidades, ou seja, o tamanho desse conjunto respeitará uma proporção de $2^{N_{\text {ponos }}}$.

\section{RESULTADOS}

As duas primeiras instâncias de testes apresentaram respostas positivas, houve a escolha do caminhão e a geração da rota para os mesmos, e não apresentam problemas como de sub-rotas, para cada rota gerada, os caminhões saem de um ponto de origem e passam pelos pontos de coleta e terminam no ponto de destino, que no caso é a indústria.

Tabela 4.1 - Resolução da Primeira Hipótese

\begin{tabular}{|c|c|c|c|c|c|c|c|c|c|}
\hline Caminhão & & & ênci & $\mathrm{col}$ & & & Carga & Capacidade & Custo/km \\
\hline 1 & 1 & 6 & 11 & 9 & 5 & 12 & 16200 & 18000 & 25 \\
\hline 2 & 1 & 3 & 8 & 2 & 12 & - & 16000 & 16000 & 28 \\
\hline 3 & - & - & - & - & - & - & 0 & 16500 & 40 \\
\hline 4 & 1 & 7 & 12 & - & - & - & 12000 & 13000 & 31 \\
\hline 5 & 1 & 4 & 10 & 12 & - & - & 8000 & 10000 & 33 \\
\hline
\end{tabular}

Fonte: Autoria Própria

Nas tabelas 4.1 e 4.2, apresentam o resultado do teste com 10 pontos e 5 caminhões e do outro teste contendo 13 pontos e 8 caminhões gerados pelo algoritmo.

Tabela 4.2 - Resolução da Segunda Hipótese

\begin{tabular}{c|ccccc|ccc}
\hline \multicolumn{1}{c}{ Caminhão } & \multicolumn{9}{c}{ Sequência da coleta } & \multicolumn{2}{c}{ Carga } & Capacidade & Custo/km \\
\hline 1 & 1 & 9 & 4 & 11 & 15 & 49 & 56 & 0.33 \\
2 & 1 & 12 & 14 & 15 & - & 34 & 41 & 0.26 \\
3 & 1 & 6 & 13 & 10 & 15 & 34 & 37 & 0.23 \\
4 & 1 & 8 & 7 & 15 & - & 29 & 33 & 0.23 \\
5 & - & - & - & - & - & 0 & 33 & 0.25 \\
6 & - & - & - & - & - & 0 & 45 & 0.33 \\
7 & - & - & - & - & - & 0 & 58 & 0.34 \\
8 & 1 & 5 & 3 & 2 & 15 & 54 & 55 & 0.31 \\
\hline
\end{tabular}

Fonte: Autoria Própria

Foi observado também que os tempos de resolução do modelo para as duas 
primeiras instâncias de testes possuem uma tendência de crescimento de forma exponencial, quando há o aumento dos pontos a serem visitados, a Tabela 4.3, mostra a relação entre quantidade de pontos e tempo de resolução para os mesmos.

Tabela 4.3 - Tempos de resolução

\begin{tabular}{ccc}
\hline Quantidade de pontos & Quantidade de Veículos & Tempo de resolução (segundos) \\
\hline 10 & 5 & 8 \\
13 & 8 & 2.290 \\
\hline
\end{tabular}

Fonte: Autoria própria

A última instância de teste com 18 pontos a serem visitados não houve a possibilidade de solução, pois a máquina de teste apresentou erro de memória RAM, de acordo com o suporte do software GUROBIC a conta para aproximação da memória estimada é feita baseada nos números de linhas e de colunas presente no modelo, os mesmos são contabilizadas a partir das seguintes fórmulas.

$$
\begin{gathered}
\text { Linhas }=\left(2^{N_{\text {ponos }}}\right) * \text { Veículos } \\
\text { Colunas }=N_{\text {pontos }} * N_{\text {pontos }} * \text { Veículos } \\
\text { Memória estimada }=\text { Linhas } * \text { Colunas } * 8 \text { bytes }
\end{gathered}
$$

Dessa forma o teste apresentou um tamanho que excedeu a capacidade de armazenamento da máquina pois de acordo com o cálculo acima seria necessário $214 \mathrm{~GB}$ de RAM, sendo assim possíveis hipóteses de instâncias de testes que apresentarem uma quantidade maior de pontos terão o mesmo resultado. Na Tabela 4.4, demonstra uma relação de possíveis novas hipóteses e o cálculo de memória RAM necessário para resolução do modelo.

Tabela 4.4 - Conferência de RAM estimada

\begin{tabular}{llllll}
\hline Hipótese & Pontos & Veículos & Linhas & Colunas & RAM (GB) \\
\hline 1 & 10 & 5 & 5.120 & 500 & 0,02 \\
2 & 10 & 8 & 8.192 & 800 & 0,05 \\
3 & 15 & 5 & 163.840 & 1.125 & 1,47 \\
4 & 15 & 8 & 262.144 & 1.800 & 3,77 \\
5 & 20 & 5 & 5.242 .880 & 2.000 & 83,89 \\
6 & 20 & 8 & 8.388 .608 & 3.200 & 214,75 \\
\hline
\end{tabular}

Fonte: Autoria própria 
Gráfico 4.1 abaixo remete a percepção em como o crescimento da memória RAM se comporta com a quantidade de pontos e veículos.

Gráfico 4.1 - Crescimento da Quantidade de RAM

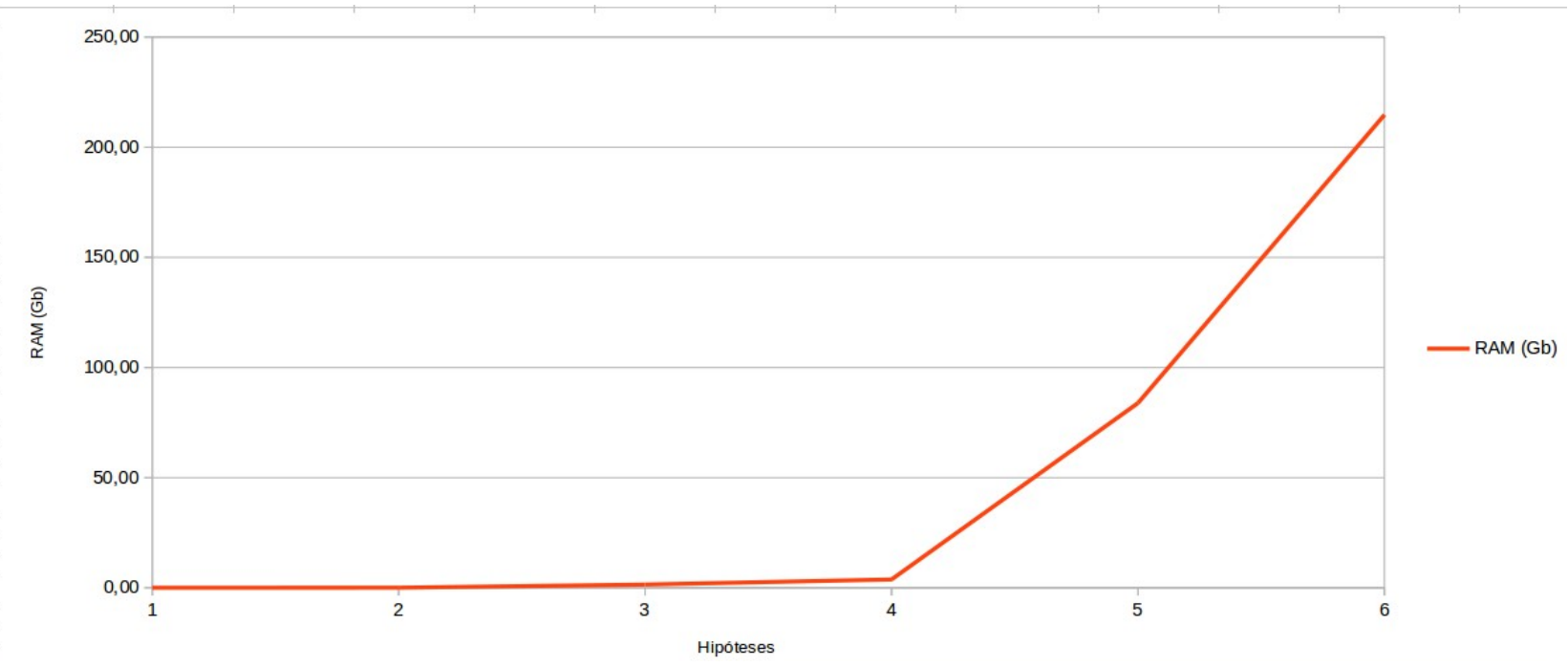

Fonte: Autoria própria

\subsection{DISCUSSÕES}

Analisando os resultados, é possível observar que o modelo tem a capacidade de atender à necessidade descrito no problema, ou seja, definir a rota para cada veículo partindo da origem, visitar todos os pontos sem gerar sub-rotas e finalizar no último ponto que é o destino definido, porém com o crescimento dos pontos a ser visitado, o modelo passa a necessitar de um tempo maior de resolução, assim como a quantidade de memória RAM presente na máquina de teste. Como os resultados obtidos pelo modelo foram apenas para 10 pontos e 13 pontos, torna-se inviável pois a limitação de pontos a serem visitados não permite a resolução de modelos maiores, ou seja, modelos que apresentarem mais pontos que nos testes sucedidos.

\section{CONCLUSÃO}

O presente trabalho abordou a adaptação do problema clássico de roteamento de veículos com a introdução da frota heterogênea com intuito da captação de produtos e entregas em um único destino. A primeira instância de teste foi para 10 pontos de coleta, com resultados satisfatórios, levando em consideração atender os critérios do modelo e o tempo computacional.

Da mesma forma a segunda instância de teste com 13 pontos de coleta, pôde-se obter resultados positivos, mesmo considerando que a diferença do tempo computacional tenha sido aproximadamente de 280 vezes maior que a primeira instância. Já a terceira instância é possível perceber tanto a limitação dos modelos lineares em problemas complexos como o de roteamento de veículos quanto a necessidade de recursos para que a máquina consiga gerar todas as combinações possíveis.

Os resultados acima demonstram que o modelo linear de $V R P$ com frota heterogênea é funcional para problemas que contenham 15 ou menos pontos de coleta, mas para problemas maiores se torna incapaz de obter a resolução. Para referências de trabalhos futuros a indicação de um método heurístico se torna imprescindível para análise dos 
resultados, levando em consideração tempo de resolução e quantidade de pontos para o estudo são fatores essenciais para o sucesso do modelo, tendo como objetivo manter o nível de serviço e buscar a minimização dos custos de operação. Somente o modelo linear não apresenta base suficiente para ser utilizado em grande escala por necessitar de um tempo computacional, processamento e armazenamento, tornando assim inviável no gerenciamento logístico no mercado, embora é possível afirmar que os resultados foram satisfatórios considerando o sucesso dos resultados, geração das rotas otimizadas.

\section{REFERÊNCIAS BIBLIOGRÁFICAS}

[1] ARAÚJO, F.; LIMA, M. A. C.; LIMA, A. A. Otimização de rota e redução dos custos logísticos: estudo de caso em uma empresa de contabilidade. [S. l.], 1 nov. 2017. Disponível em: http://www.brjd.com.br/index.php/BRJD/article/view/109. Acesso em: 15 mar. 2019.

[2] ARENALES, M.; ARMENTANO, V. A.; MORABITO, R.; YANASSE, H. H. Pesquisa operacional. Rio de Janeiro: Campus/elsevier, 2007. 523 p. ISBN 10-85352-145-1454-2

[3] BALLOU, R.H. Gerenciamento da Cadeia de Suprimentos / Logística Empresarial. 5ed. Ed. Bookman, São Paulo, 2006.

[4] BeRTAGLiA, P. Logística e Gerenciamento da Cadeia de Abastecimento. 2.ed. Editora Saraiva - 2003.

[5] BOWERSOX, D.; CLOSS, D. Logística Empresarial: O processo de Integração da Cadeia de Suprimento. 1.ed. -Editora Atlas S.A - 2007.

[6] MENDONÇA, A. A. A. Modelos e técnicas de local branching para o problema de abastecimento de linhas de montagem. Dissertação (Mestrado). Universidade Federal de Minas Gerais - UFMG, 2011.

[7] OLIVEIRA, E.S. A abordagem da pesquisa operacional aplicada à gestão de materiais e a logística: contribuição para o ensino do modelo de programação linear em dois níveis. Dissertação (Mestrado). Universidade Estadual do Norte Fluminense - UENF, 2005.

[8] PICININ, C. T.; KOVALESKI, J. L. Sistema logístico e a tendência para empresas prestadoras de serviços em logística. XXIX Encontro Nacional De Engenharia De Produção - ENEGEP, ABEPRO, vol. 1, p. 1-15, 2009.

[9] SUPPLY CHAIN MANAGEMENT AND ITS RELATIONSHIP TO LOGISTICS, MARKETING, PRODUCTION, AND OPERATIONS

MANAGEMENT. Tennessee: Journal of Business Logistics, 2008, 2008- . 20/04/2019. Disponível em:

https://www.academia.edu/32585140/Supply_Chain_Management_and_Its_Relationsh ip_to_Logistics_Marketing_Production_and_Operations_Management. Acesso em: 20 abr. 2019.

[10] TAHA, Hamdy A. (2008) Pesquisa operacional: uma visão geral / Hamdy A. Taha; tradução Arlete Simille Marques; revisão técnica Rodrigo Arnaldo Scarpel. - São

Paulo: Pearson Prentice Hall. 